\title{
O tema da saúde no Senado Federal: uma análise dos projetos de lei de 2011-2012 ${ }^{1}$
}

The subject of health in the Brazilian Federal Senate: an analysis of draft law presented in 2011-2012

\section{Evelyn Britto}

Graduanda em Saúde Coletiva. Bolsista da Fundação Oswaldo Cruz. Brasília, Brasil.

\section{Maria Célia Delduque}

Advogada, Doutora em Saúde Pública, Pesquisadora em Direito Sanitário da Fundação Oswaldo Cruz e da Universidade de Brasília. Brasil.

Resumo: $\mathrm{O}$ artigo analisa os projetos de lei, na área de saúde, iniciadas pelo Senado Federal nas duas primeiras sessões legislativas da 54른 Legislatura. Trata-se de uma pesquisa descritivo-analítica com o objetivo de descrever os partidos políticos propositores de matérias na área da saúde no Senado Federal brasileiro, a região de origem dos propositores e os tipos de proposições apresentadas, seu conteúdo material. Nota-se mudança no perfil dos senadores e nas temáticas apresentadas quando comparados a período anterior, além de uma ampliação do número de proposições em matéria sanitária.

Palavras-chave: Senado Federal, Brasil; legislação em saúde; Direito Sanitário.

Abstract: This paper analyses the draft bills on health initiated in the Brazilian Federal Senate in the first two legislative sessions of the 54th Legislature. This is a descriptive analytic research with the aim of describing the political parties, the region of origin of the proponents and the types of propositions, their matters. There are some changes in the profile of senators and themes presented when compared to previous period, and an expansion in the number of propositions on health issues.

Keywords: Federal Senate, Brazil; health legislation; Health Law.

Resumen: El artículo examina los proyectos de ley sobre materias de salud iniciados en el Senado Federal de Brasil en las dos primeras sesiones legislativas de la 54a Legislatura. Se trata de una investigación descriptiva analítica con el objetivo de describir los partidos políticos y la región de origen de los autores de proposiciones en materia de salud, el tipo de proposiciones y su contenido material. Observa se un cambio en el perfil de los senadores y en los temas presentados en comparación con el período anterior, además de la expansión del número de proposiciones sobre temas de salud.

Palabras Ilave: Senado Federal, Brasil; legislación de salud; Derecho Sanitario

\footnotetext{
${ }^{1}$ Pesquisa fruto da bolsa PIBIC/CNPq/FIOCRUZ- 2012/2013.
} 


\section{Introdução}

O direito à saúde não se efetiva somente por respaldo constitucional, mas também por normas infraconstitucionais elaboradas pelo Poder Legislativo para auxiliar na implementação de políticas públicas pelo Estado.

Questões afetas à saúde têm originado vários debates e discussões no Congresso Nacional brasileiro, principalmente depois da promulgação da Carta Política de 1988, com temas como a efetivação do Sistema Único de Saúde (SUS), sua regulamentação por meio da Lei Orgânica da Saúde (LOS), a atuação das agências reguladoras, a vigilância sanitária e ambiental, drogas, saúde indígena etc.

Porém, observa-se hodiernamente, apesar da importância e valorização do Legislativo pela Carta Política brasileira, que há uma crise democrática de representatividade, pois, no decorrer do processo de consolidação institucional, o Poder Executivo é o que mais legisla, principalmente pelo mecanismo de medidas provisórias e também pelo fenômeno identificado como judicialização da política (Barroso, 2008), em que são debatidos no Supremo Tribunal Federal os principais temas da Nação, fazendo com que o Legislativo sofra uma atrofia em sua atuação. Isso, certamente, dá origem a consequências sobre o projeto sanitário proposto pela Constituição de 1988.

O presente artigo é o resultado da análise do resultado de dois fatores proporcionados pelo constitucionalismo dirigente brasileiro de 1988: 1) a ratificação do direito à saúde como direito de todos e dever do Estado; e 2) a retomada da importância do Poder Legislativo - na definição clássica da teoria da separação dos poderes de Montesquieu - especificamente o Senado Federal.

O campo de análise é delimitado basicamente em dois aspectos: primeiramente, na atuação legislativa sanitária no Senado Federal, porque é a Casa do Federalismo onde vige a horizontalidade de representação e porque, teoricamente, seria a casa mais analítica do Congresso, conforme seu caráter de ultima ratio no desenvolvimento das atividades legislativas. Em segundo lugar, na delimitação temporal, buscou-se analisar as duas primeiras sessões 
legislativas ordinárias da 54ª Legislatura (2011-2012), pela sua atualidade para o campo sanitário.

Tem-se, assim, o objetivo de identificar os partidos políticos e a região de origem dos propositores de matérias na área da saúde no Senado Federal, e os tipos de proposições apresentadas e seu conteúdo material.

\section{Metodologia}

Tratou-se de uma pesquisa descritivo-analítica, cuja base de dados é secundária, consubstanciada no sítio eletrônico Observatório da Saúde no Legislativo (OLS), hospedado no servidor da Diretoria Regional de Brasília da Fundação Oswaldo Cruz.

O OSL descreve cada projeto com detalhe por categorias temáticas, informando ano original, partido e unidade federada do propositor, e estado da tramitação, entre outras informações. São mais de trinta categorias especificadas, abordando os temas mais relevantes da saúde.

Para facilitar a compreensão dos assuntos tratados, foram definidos grandes grupos temáticos baseado nas delimitações feitas por Romero et al. (2000) e na identificação de novas matérias de saúde. As categorias que apresentaram frequência muito baixa e não se enquadraram nos descritores definidos foram agrupadas e consideradas como "outros".

A caracterização dos senadores propositores foi realizada a partir do levantamento dos dados informados na página oficial de cada senador na internet. Foram levantados: profissão, região de origem e orientação ideológico-partidário de filiação. No caso de haver mais de uma profissão informada, adotou-se a primeira. A ocupação dos senadores, fora do âmbito da política, foi considerada como a profissão informada que, muitas vezes, não coincide com a formação acadêmica.

Em relação à caracterização da orientação ideológica e partidária dos senadores foi utilizada a classificação utilizada por Romero et al. (2000) considerando a existência de novos partidos e os posicionamentos políticos habitualmente adotados.

Para a coleta do conteúdo material dos projetos de lei do Senado, foram levantados os textos iniciais - denominados de 'avulsos' - disponibilizados na 
página eletrônica do Senado Federal, por meio de análise simplificada de todas as justificativas expostas nos projetos.

O estudo computou somente os projetos de lei iniciados no Senado Federal e apresentados no período de análise (2011 e 2012). A escolha pela análise de projetos de lei (PLC) se deu por representar a via legislativa mais utilizada (Gomes, 2012).

Foram considerados os projetos disponibilizados pelo OSL até o mês de setembro de 2012.

\section{Resultados}

Os resultados compreendem o período de 20 meses (de fevereiro de 2011 até setembro de 2012). Foram apresentados 126 projetos de lei iniciados no Senado Federal brasileiro relacionados à temática de saúde pública. 93 projetos no ano de 2011 e 33 em 2012. Dessa forma, 73,8\% dos projetos corresponderam ao ano de 2011.

Destaca-se que dois desses projetos foram iniciados por comissões, não descrevendo região de origem, profissão e partido político. Dos 81 senadores que exerceram mandato no período de estudo, somente 37 (46\%) atuaram com a proposição de, pelo menos, um projeto na área de saúde.

Os senadores representantes das regiões do Nordeste e Centro-Oeste se destacaram na produção de projetos de lei. A atuação dos senadores das regiões Sul e Sudeste neste assunto é pouco frequente (Tabela 1).

Tabela 1 - Projetos de Lei do Senado segundo regiões de origem do propositor. Senado Federal, Brasil, 2011-2012

\begin{tabular}{lcc}
\hline \multicolumn{1}{c}{ Região } & Quantidade & $\%$ \\
\hline Norte & 19 & 15,2 \\
Nordeste & 57 & 47,2 \\
Centro-Oeste & 24 & 19,2 \\
Sudeste & 14 & 11,2 \\
Sul & 10 & 8,0 \\
\hline Total & 124 & 100,0 \\
\hline
\end{tabular}

Fonte: Observatório da Saúde no Legislativo

Os representantes do Partido do Movimento Democrático Brasileiro (PMDB) e Partido dos Trabalhadores (PT) são os mais numerosos, com 19 e 
12 senadores respectivamente. Entretanto, quando é analisada a frequência de produção dos ideológicos partidários, os socialistas e comunistas são os mais atuantes $(47,6 \%)$, seguido dos liberas e direita clássica (29,8\%). Em geral, os assuntos sobre políticas públicas e controle de doenças foram os mais explorados pelas orientações ideológicas de esquerda. Senadores filiados aos partidos socialistas e comunistas tiveram grande participação nos temas de medicamentos, vacinas, insumos e produtos para a saúde.

Tabela 2 - Projetos de Lei do Senado segundo orientação ideológica do propositor. Senado Federal, Brasil, 2011-2012.

\begin{tabular}{l|c|c}
\hline \multicolumn{1}{c|}{ Orientação ideológico-partidária } & Quantidade & $\%$ \\
\hline Socialistas e comunistas & 52 & 41,9 \\
Ecologistas & 7 & 5,6 \\
Trabalhistas & 11 & 8,9 \\
Centristas & 17 & 13,7 \\
Liberais e direita clássica & 37 & 29,8 \\
\hline Total & 124 & 100,0 \\
\hline
\end{tabular}

Fonte: Observatório da Saúde no Legislativo

Os senadores médicos se destacaram como os principais atuantes na área de saúde. A afinidade da profissão com a área de conhecimento mostra a predominância de atuação. Os professores e empresários apareceram com grande atividade na apresentação de proposições de projetos com 13,7\% e $8,9 \%$, respectivamente.

Os temas mais presentes nos textos legislativos no Senado Federal foram políticas públicas de saúde com $29,4 \%$ dos projetos, seguido por controle de doenças com $24,6 \%$ e medicamentos, vacinas, insumos, produtos para a saúde com $21,4 \%$. Projetos a respeito da saúde do idoso, indígena e bucal foram os de menores frequências dentro das políticas de saúde pública específica $(0,8 \%)$ (Tabela 3$)$.

Se os temas de saúde dos projetos de lei forem distribuídos conforme a profissão médica que foi predominante, observa-se que os assuntos mais tratados nos discursos de médicos são em sua maioria, sobre políticas públicas de saúde, controle de doenças e saúde da criança e adolescente. 
Em um esquema comparativo quanto a relevância dos temas propostos nas sessões legislativas 1995-1996, no trabalho conduzido por Romero et al. (2000) e o presente, verifica-se uma maior ênfase no controle de doenças, enquanto a saúde do trabalhador e a saúde mental, que no passado foram objetos de relevância para o Senado, perderam em importância na atual legislatura.

A saúde reprodutiva, saúde do idoso e transplantes que foram preocupação dos senadores há mais de uma década, não foram identificados como temas prioritários, na pesquisa, enquanto que o medicamento foi encontrado em projetos de lei, apenas na atualidade.

Tabela 3 - Projetos de lei segundo grupos temáticos. Senado Federal, Brasil, 2011-2012.

\begin{tabular}{l|r|r}
\hline \multicolumn{1}{c|}{ Temas } & n & $\%$ \\
\hline Políticas Públicas de Saúde & 37 & 29,37 \\
Controle de doenças & 31 & 24,60 \\
Medicamentos, vacinas, insumos, produtos para a saúde & 27 & 21,43 \\
Indenizações, isenções de imposto, benefícios & 19 & 15,08 \\
Drogas & 19 & 15,08 \\
Vigilância Sanitária & 16 & 12,70 \\
Saúde Suplementar & 14 & 11,11 \\
Regulamentação das profissões na área de saúde & 11 & 8,73 \\
Saúde da criança e adolescente & 12 & 9,52 \\
Saúde de pessoas com deficiência & 10 & 7,94 \\
Saúde de grupos populacionais específicos & 9 & 7,14 \\
Alimentação e Nutrição & 9 & 7,14 \\
Vigilância Epidemiológica e Ambiental & 8 & 6,35 \\
Causas Externas & 8 & 6,35 \\
Propaganda & 8 & 6,35 \\
Saúde Mental & 7 & 5,56 \\
Saúde do Trabalhador & 7 & 5,56 \\
Saúde reprodutiva & 6 & 4,76 \\
Saneamento & 5 & 3,97 \\
Transplantes & 3 & 2,38 \\
Saúde do Idoso & 1 & 0,79 \\
Saúde Bucal & 1 & 0,79 \\
Saúde da Mulher & 5 & 3,97 \\
Saúde Indígena & 1 & 0,79 \\
Outros & 9 & 7,14 \\
\hline Total & $\mathbf{2 8 3}$ & $\mathbf{2 2 4 , 6 0}$ \\
\hline
\end{tabular}


Fonte: Dados coletados no Observatório da Saúde no Legislativo

Quadro 1 - Evolução de temas de projetos de lei do Senado, 1995-6/2011-12 Brasil

\begin{tabular}{|c|c|c|c|}
\hline Posição & $\begin{array}{c}\text { ranking } \\
1995-1996\end{array}$ & Evolução & $\begin{array}{c}\text { ranking } \\
2011-2012\end{array}$ \\
\hline $1^{\circ}$ & \multicolumn{3}{|c|}{ Drogas Políticas Públicas } \\
\hline $2^{\circ}$ & \multicolumn{3}{|c|}{ Políticas Públicas, Controle de doenças } \\
\hline $3^{\circ}$ & \multicolumn{3}{|c|}{$\begin{array}{c}\text { Regulamentação das profissões na área } \\
\text { de saúde }\end{array}$} \\
\hline $4^{\circ}$ & \multicolumn{3}{|c|}{ Planos e Seguros $\quad$ Drogas / Indenizações } \\
\hline $5^{\circ}$ & \multicolumn{3}{|c|}{ Vigilancia Sanitária $\longmapsto$ Vigilância Sanitária } \\
\hline $6^{\circ}$ & \multicolumn{3}{|c|}{ Transplantes $\quad$ Saúde Suplementar } \\
\hline $7^{\circ}$ & Saúde do Trabalhador & & ão das profissões na área de \\
\hline $8^{\circ}$ & \multicolumn{3}{|r|}{ ça e do adolescente } \\
\hline $9^{\circ}$ & \multicolumn{3}{|l|}{ Saúde do Idoso } \\
\hline $10^{\circ}$ & \multicolumn{3}{|l|}{ Saúde reprodutiva } \\
\hline $11^{\circ}$ & \multicolumn{3}{|l|}{ Alimentação e Nutrição * } \\
\hline $12^{\circ}$ & \multicolumn{3}{|l|}{ Saúde da criança e do adolescente } \\
\hline 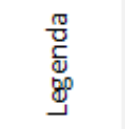 & \multicolumn{3}{|c|}{$\longmapsto$ manteve a posição $\quad$ caiu ou saiu do ranking $\quad \longmapsto$ subiu ou entrou no ranking } \\
\hline
\end{tabular}

Fonte: Romero et al.(2000) e Observatório da Saúde no Legislativo

\section{Discussão}

O primeiro ano analisado, 2011, teve praticamente o dobro de projetos apresentados quando comparado ao ano subsequente. A maior produção legislativa coincide com a posse dos senadores, que segundo Delduque e Marques (2012): "É o momento de dar ao eleitor uma satisfação e propor projetos de lei, especialmente de caráter social, notadamente na área da saúde, área de maior visibilidade junto à população". O Gráfico 1 ilustra a distribuição temporal da proposição de projetos de lei tratando de matérias de saúde em cada ano analisado, confirmando que, no segundo ano da legislatura o número de proposições apresentou diminuição considerável se comparada ao primeiro.

Durante o período de análise, nenhum projeto se transformou em lei. Um projeto foi arquivado e os demais mantiveram-se em tramitação. 
No Regimento das casas legislativas, não são estabelecidos prazos para os projetos de lei de iniciativa parlamentar serem analisados, ficando o ritmo da tramitação à mercê do interesse político na matéria. $O$ processo legislativo de análise e votação de um projeto de lei é muito lento. O tempo médio de tramitação constatado no recorte do estudo foi de aproximadamente 12 meses.

Gráfico 1 - Projetos de lei segundo o mês de apresentação. Senado Federal, Brasil, 2011-2012.

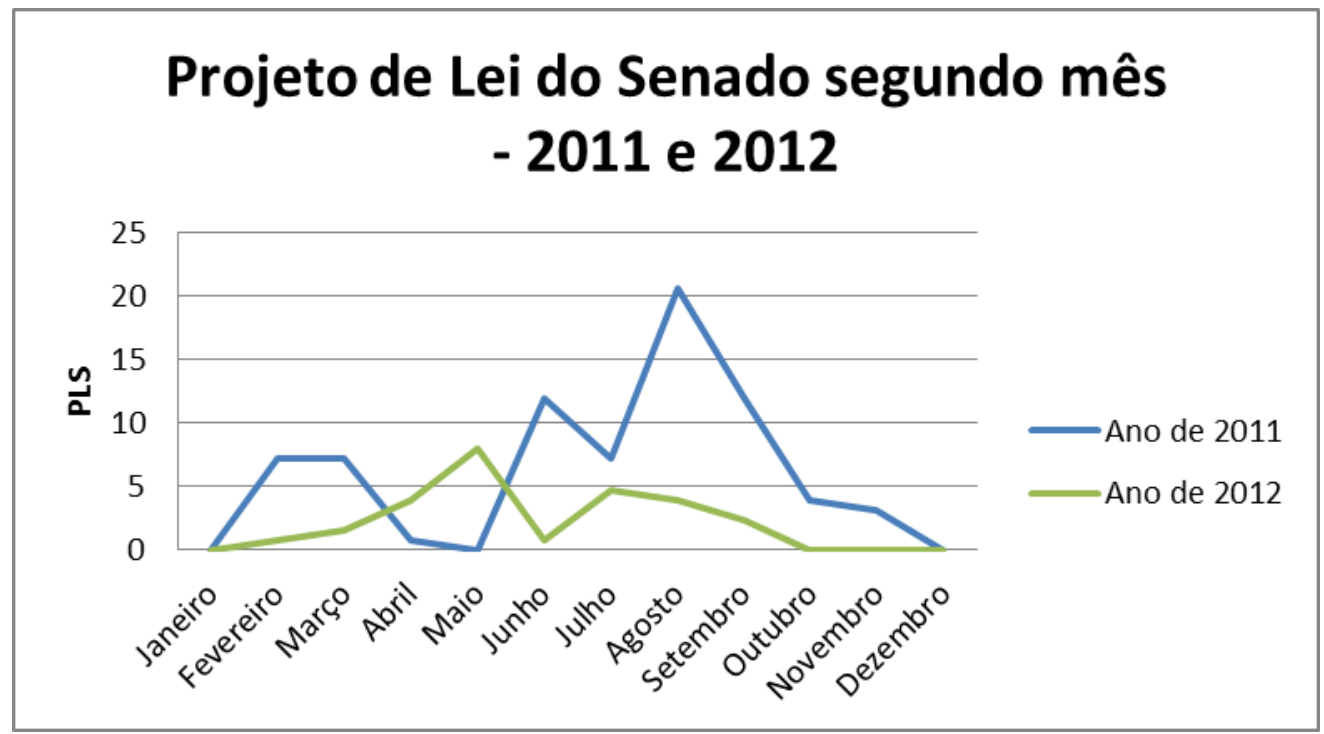

Fonte: Observatório da Saúde no Legislativo

O perfil dos senadores propositores de matérias de saúde pública alterou-se, quando comparado ao das sessões legislativas de 1995 e 1996, nos estudos de Romero et al. (2000). Neste estudo, observou-se um significativo aumento na produção dos representantes da Região Centro-Oeste, o que não se vislumbra no estudo passado. Outra mudança encontrada é no perfil partidário dos senadores mais atuantes. No estudo anterior apresentavam-se os liberais, enquanto neste, apresentam-se, majoritariamente, os senadores socialistas e comunistas. Esse dado reflete a influência dos governos no poder, na atuação dos senadores.

Em relação às profissões, os médicos permanecem como os mais ativos na elaboração de propostas em saúde, constatando a relevância da profissão no interesse do senador em atuar em área de saúde. 
As temáticas também se alteraram, com exceção das proposições que tratam de vigilância sanitária. Novos assuntos emergiram e dominaram 0 ranking como, por exemplo, a maior preocupação com assistência farmacêutica, medicamentos, vacinas e insumos, e os grandes gastos econômicos que a saúde está trazendo aos cidadãos, respaldada em indenizações, isenções de impostos e benefícios.

O expressivo aumento do grupo temático 'controle de doenças' deve ser destacado, pois nota-se que houve maior enfoque em questões de prevenção. As justificativas dos projetos descreviam a importância de atuar nos níveis de prevenção, identificando-os como forte aliado na redução de custos e melhores condições de vida.

Os problemas de saúde pública, na visão dos senadores, ganham outros enfoques, caindo de posição o tema de 'drogas' e ganhando destaque o tema 'políticas públicas de saúde'. Interessante notar que apesar de a judicialização ser um tema bastante discutido, não houve, por parte dos senadores, qualquer intenção de proposição legislativa no sentido de minorar esse fenômeno social que vem desequilibrando as contas do Sistema Único de Saúde. O tema dos 'planos e seguros de saúde', além de cair no ranking de prioridades, em comparação a 1995-1996, passou a ser tratado no âmbito do Senado Federal pela denominação constitucional de 'saúde suplementar'.

Analisar os projetos de lei permite compreender a visão da casa revisora frente às políticas de saúde. Esses resultados trazem à luz que os grandes problemas de saúde levados à consideração do Senado Federal brasileiro naquele período são relativos aos temas 'políticas públicas de saúde', 'controle de doenças', 'medicamentos', 'drogas' e 'indenizações'.

A característica desse ator social que produz o arcabouço legal na área de saúde é ser predominantemente de formação médica, representante da Região Norte e vinculado a partidos de ideologia socialista e/ou comunista.

Nota-se um incremento no número de proposições em matéria sanitária (168\%), utilizando como parâmetro a quantidade de projetos de lei do Senado apresentados nos anos de 1995 e 1996 (Romero, 2000), sem, no entanto, haver uma profusão de novas leis na área de saúde advindas desses mesmos projetos de lei de iniciativa parlamentar. 
Outro destaque é a mudança do perfil dos senadores ao longo dos anos e o aparecimento de outras temáticas com grande expressão como, por exemplo, o controle de doenças. Essas mudanças dos projetos de lei podem ser justificadas pela valorização da concepção preventiva, essa um movimento mundial.

\section{Conclusões}

O papel do Poder Legislativo no cenário estatal é indubitavelmente necessário. No entanto, quando se observa a produção legislativa em saúde, em especial as proposições de iniciativa parlamentar, vê-se que o que é produzido não tem sido efetivamente se transformado em lei, embora muitas vezes tenha significativa importância para o campo da saúde.

O Observatório da Saúde no Legislativo e ainda outros meios virtuais de informação legislativa são instrumentos preciosos para o acompanhamento do que é produzido em matéria legislativa e, igualmente, da atuação parlamentar.

O alinhamento dos Poderes Executivo e Legislativo na formulação da política pública de saúde é essencial para que ela possa existir, pois é da fonte legislativa - inclusive da lei orçamentária - que a política pública de saúde pode ganhar existência concreta e ser plenamente executada.

O acompanhamento sistemático da produção legislativa em saúde é uma prática importante para aquele que deseja estudar e investigar as políticas públicas, especialmente na seara social. O Senado Federal, na qualidade de casa revisora e representante dos entes federados tem o importante papel de ser a caixa de ressonância dos anseios nacionais e a observação de sua produção legislativa em saúde é fundamental para quem acompanha as políticas públicas sanitárias.

\section{Referências}

BARROSO, Luís Roberto. Interpretação e Aplicação da Constituição. São Paulo: Saraiva, 2008. ISBN 8502049291.

DELDUQUE, Maria Célia; MARQUES, Silvia Badim. A produção legislativa em saúde entre 2007 e 2010: resultados preliminares do Observatório da Saúde no Legislativo. In: DELDUQUE, Maria Célia \& ROMERO, Luiz Carlos. Produção normativa em saúde: políticas setoriais e regulação. Brasília: Senado Federal, 2012, pp. 295-302. ISBN 978-85-7018-462-7.

GOMES, Fábio de Barros Correia. Características da tramitação de projetos de lei e de propostas de emenda à Constituição em matérias de saúde na Câmara 
dos Deputados entre 1999 e 2006. In: In: DELDUQUE, Maria Célia \& ROMERO, Luiz Carlos. Produção normativa em saúde: políticas setoriais e regulação. Brasília: Senado Federal, 2012, pp. 241-260. ISBN 978-85-7018462-7.

ROMERO, Luiz Carlos et al. Atividades do Senado Federal brasileiro na área da saúde pública, 1995 e 1996. Revista Panamericana de Salud Pública, 7(2):69-78, fev. 2000. ISSN 1020-4989.

Recebido para publicação em 17 de março de 2014. Admitido para publicação em 26 de novembro de 2014. 